\title{
HIGH-RESOLUTION ISOCAM VIEW OF NUCLEAR AND CIRCUMNUCLEAR STARBURSTS IN BARRED GALAXIES
}

\author{
D. FRIEDLI \\ Université Laval and OMM, Québec, QC, G1K 7P4, Canada
}

H. WOZNIAK

Observatoire de Marseille, F-13248 Marseille cedex 4, France AND

L. MARTINET, D. PFENNIGER

Observatoire de Genève, CH-1290 Sauverny, Switzerland

\section{Introduction}

Non-axisymmetric gravitational potentials are likely to play an important role in generating central activity in galaxies, i.e. starbursts and/or active galactic nuclei (AGN). For instance, the often observed secondary bars embedded in larger, primary, ones (Friedli \& Martinet 1993; Wozniak et al. 1995; Friedli et al. 1996) might play a key role in feeding AGN, by accreting gas down to the centre at scales of a few parsecs. On the other hand, the frequent presence of a nuclear ring of star formation located between both bars indicates that double-barred galaxies are intimately linked to the formation of some circumnuclear starbursts. This type of galaxies might give useful clues to the connection between starbursts and AGN.

Below, as part of a larger sample of nearby barred galaxies (Wozniak et al. 1997a) being taken by ISOCAM ${ }^{1}$ on board of the ISO satellite, we briefly discuss the characteristics of the circumnuclear mid-infrared (MIR) emission of two double-barred galaxies, namely NGC 4321 (M100) and NGC 1097. The observations were done using LW2 $(5-8.5 \mu \mathrm{m})$ and LW3 $(12-18 \mu \mathrm{m})$ broad band filters at the $1.5^{\prime \prime} \mathrm{px}^{-1}$ PFOV.

${ }^{1}$ This work is based on observations with ISO, an ESA project with instruments funded by ESA Member States (especially the PI countries: France, Germany, the Netherlands and the United Kingdom) and with the participation of ISAS and NASA. 


\section{ISOCAM Observations of NGC 4321 and NGC 1097}

NGC 4321 (M100) is a Virgo giant moderately barred spiral galaxy with mild star formation (SFR $\approx 5 \mathrm{M}_{\odot} \mathrm{yr}^{-1}$ ). Detailed multi-wavelength studies indicate a very intricate central morphology (Knapen et al. 1995; Wozniak et al. 1997b). Secondary and primary bars are aligned. The main MIR features are two prominent peaks near secondary bar ends (Wozniak et al. $1997 \mathrm{a}, \mathrm{b})$. The brightest spot emits $\approx 1.4 \mathrm{mJy} \operatorname{arcsec}^{-2}$ in LW2. The ratio LW2/LW3 $\approx 1.1-1.2$ which might suggest the presence of WC stars.

NGC 1097 is a strongly barred LINER galaxy which hosts a powerful starburst ( $\mathrm{SFR} \approx 22 \mathrm{M}_{\odot} \mathrm{yr}^{-1}$ ). Secondary and primary bars are misaligned by $\approx-63^{\circ}$ (Friedli et al. 1996). Like in $\mathrm{K}$, the $\mathrm{H} \alpha$ circumnuclear ring (CNR) is composed of tight trailing spirals (Storchi-Bergman et al. 1996). Along the CNR, six distinct MIR peaks are distributed two by two with respect to central symmetry. Two of them are close to secondary bar ends, while two other (brightest and weakest ones) are nearly aligned with the primary bar. There seems to be no tight correlations with $\mathrm{H} \alpha$ and $\mathrm{K}$ emissions. They all have LW2/LW3 < 1.0; highest values are found between the MIR peaks! The brightest spot is roughly 3 times more intense than in NGC 4321.

\section{Discussion}

The CNRs of NGC 4321 and NGC 1097 show distinct MIR morphologies, various MIR intensities, and LW2/LW3 color. These features can be interpreted by differences in SFRs, age of the starbursting regions, and central dynamics. i) Young starbursts $(<3-5 \mathrm{Myr})$ contain several compact star clusters which lead to NIR and MIR spots due to OB and WR stars associated to hot dust responsible for the background. Old starbursts ( $>5-7 \mathrm{Myr}$ ) display more scattered distributions of stars and dust. NIR and MIR peaks are thus much less intense or have disappeared. ii) NGC 4321 is generally interpreted in the classical framework of one single bar with two ILRs, i.e. both bars are supposed to rotate with the same pattern. On the contrary, in NGC 1097 the secondary bar trails the primary one which indicates the existence of two dynamically decoupled bars (Friedli \& Martinet 1993).

\section{References}

Friedli D., Martinet L., 1993, A\&A 277, 27

Friedli D., Wozniak H., Rieke M., Martinet L., Bratschi P., 1996, A\&AS 118, 461

Knapen J.H., Beckman J.E., Heller C.H., Shlosman I., de Jong R.S., 1995, ApJ 454, 623

Storchi-Bergmann T., Wilson A.S., Baldwin J.A., 1996, ApJ 460, 252

Wozniak H., Friedli D., Martinet L., Martin P., Bratschi P., 1995, A\&AS 111, 115

Wozniak H., Friedli D., Martinet L., Pfenniger D., 1997a, in Extragalactic Astronomy in the Infrared, G.A. Mamon et al. (eds.), in press (see also astro-ph/9708137)

Wozniak H., Friedli D., Martinet L., Pfenniger D., 1997b, A\&A, in press 